\title{
Large stroke fast steering mirror for free-space optical communication
}

Gérald Aigouy, Adrien Guignabert, Etienne Betsch, Clément Cote, Jocelyn Rebufa, et al.

Gérald Aigouy, Adrien Guignabert, Etienne Betsch, Clément Cote, Jocelyn Rebufa, Xavier De Lepine, Marc Fournier, Sylvain Duc, Olivier Sosnicki, Pierre Personnat, Frank Claeyssen, "Large stroke fast steering mirror for freespace optical communication," Proc. SPIE 11852, International Conference on Space Optics - ICSO 2020, 1185250 (11 June 2021); doi: $10.1117 / 12.2599967$

SPIE Event: International Conference on Space Optics - ICSO 2021, 2021, Online Only 


\section{International Conference on Space Optics-ICSO 2020}

Virtual Conference

30 March-2 April 2021

Edited by Bruno Cugny, Zoran Sodnik, and Nikos Karafolas
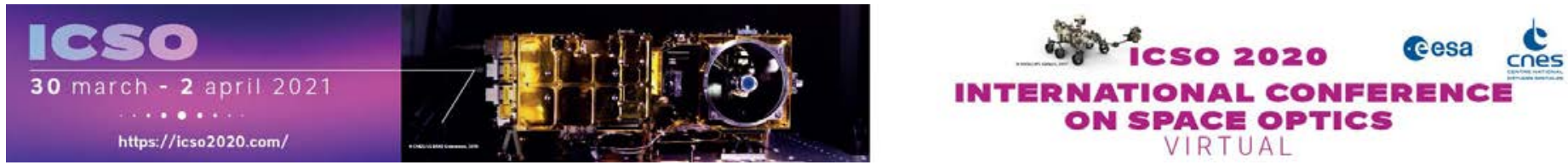

\section{Large stroke fast steering mirror for free-space optical communication}

\section{Cesa issopoceatings lecnes}




\title{
LARGE STROKE FAST STEERING MIRROR FOR FREE-SPACE OPTICAL COMMUNICATION
}

\author{
Gérald Aigouy, Adrien Guignabert, Etienne Betsch, Clément Cote, Jocelyn Rebufa, Xavier De \\ Lepine, Marc Fournier, Sylvain Duc, Olivier Sosnicki, Pierre Personnat, Frank Claeyssen,
}

\begin{abstract}
CEDRAT TECHNOLOGIES, 59 Chemin du Vieux Chêne - Inovallée - 38246 MEYLAN Cedex France
\end{abstract}

\begin{abstract}
In order to cover new needs of large stroke beyond $+/ 2^{\circ}$ for Free-space optical communication (FSO), inter-satellite links in constellations, as well as for Deep Space Optical Communications (DSOC), CEDRAT TECHNOLOGIES (CTEC) has developed a compact, low-power, high-stroke and high-bandwidth, Fast Steering Mirrors called M-FSM.

The proposed M-FSM design is based on proprietary MICA ${ }^{\mathrm{TM}}$ flexure bearing magnetic actuators technology, taking heritage from other MICA ${ }^{\mathrm{TM}}$ Space application domains, such as long-life space cryogenic refrigerators. The M-FSM includes a dedicated 30mm silicon carbide (SIC) mirror developed by CTEC, based on PAM30 Flight Model knowhow heritage, currently under manufacturing for NASA PYSCHE Deep Space Optical Communication (DSOC) spacecraft.
\end{abstract}

Keywords: Fast Steering Mirror; Point Ahead Mechanism; Beam Steering Mirror; Fine Steering Mirror; Optical Pointing; Optical Communication; Laser Communication; Deep Space Optical Communication; Tip-Tilt Mirror Mechanism; Magnetic Mirror Steering Mechanism; Inter-satellite links; Giant Constellations.

\section{INTRODUCTION}

Low-power high-stroke high-bandwidth Fast Steering Mirrors (FSM) are demanded for Free-Space Optical communication (FSO) in future LEO constellations inter-satellite links as well as in Deep Space Optical Communications (DSOC).

For 20 years, CTEC has provided piezoelectrically-actuated Fast or Fine Steering Mirrors (FSM) for space missions and optronics [1,2]. In 2020, CTEC has delivered the Flight Models of the FSM required for PSYCHE DSOC mission of NASA JPL [3]. These FSM named PAM30 are Tip-tilt mechanisms based on 4 APA®. They offer a large bandwidth (> $2 \mathrm{kHz}$ ) and fine resolution, but angular strokes are limited to $+/-2.8 \mathrm{mrad}$ (Figure1).

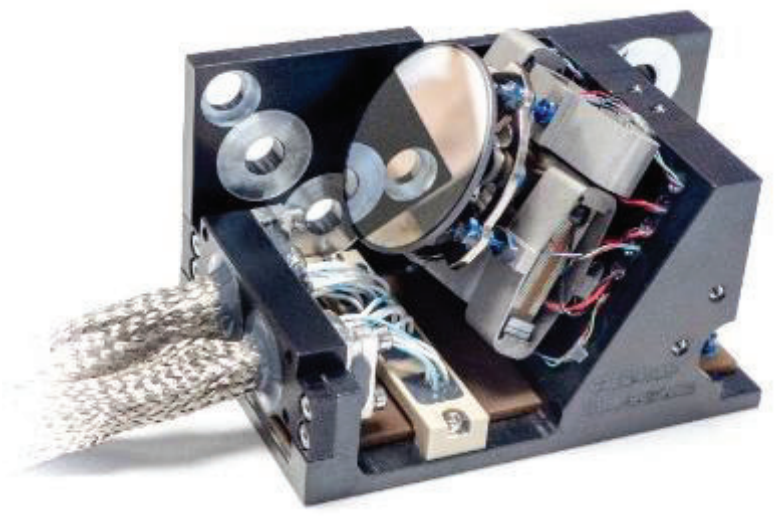

Figure 1. PAM30 for NASA JPL PSYCHE Mission 
As such small strokes are constraining for optical and control design for FSO optical terminals, CTEC has developed a new FSM based on magnetic actuation called M-FSM62 (Figure 2). This targets larger tilt stroke, larger than $+/-2$ mechanical degrees (+/-35mrad), low power, low heating and high bandwidth: $200 \mathrm{~Hz}$ full stroke and $1 \mathrm{kHz}$ at low level. At first a Breadboard Model (BBM) was realised using a $\square 10 \mathrm{~mm}$ glass mirror. Then an Engineering Model (EM) has been developed [4]. It steers a $\mathrm{SiC}$ mirror with a $\square 31 \mathrm{~mm}$ diameter using a better mirror support, keeping the same magnetic actuation design.

The paper will review the technology of the M-FSM62 as well as its performances.

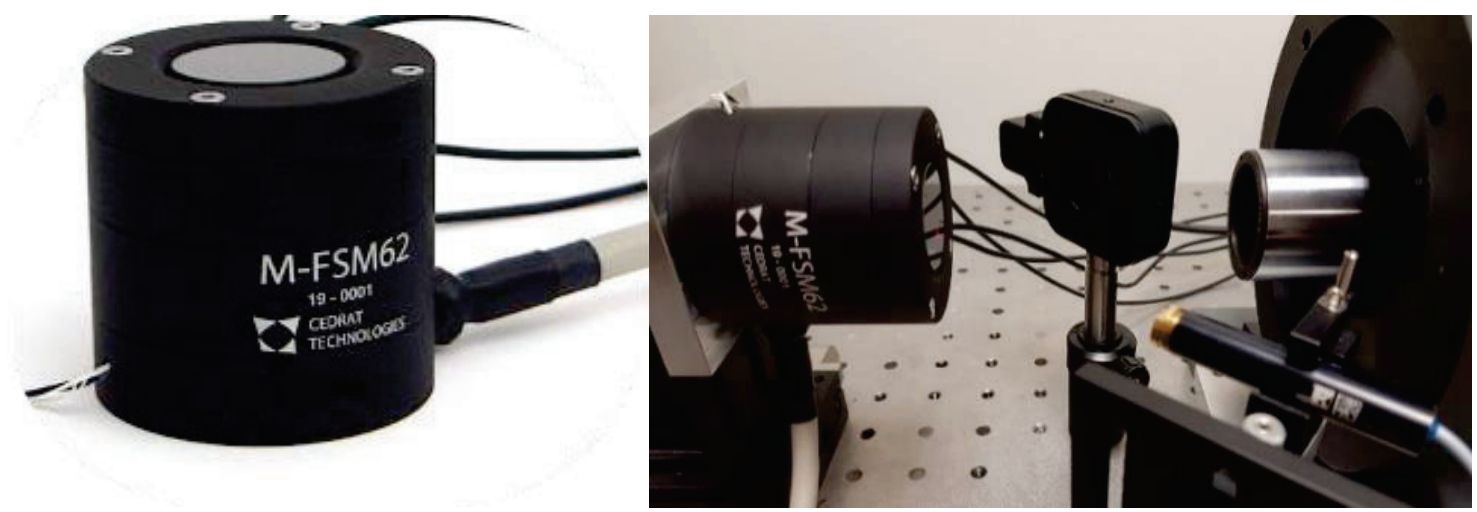

Figure 2. M-FSM62 EM on optical test bench

\section{M-FSM TECHNOLOGY}

The most conventional magnetically actuated FSMs for space rely on Voice Coil Motors using Lorentz forces [5].

Although CTEC manufactures also Voice Coil actuators for space applications, another type of magnetic actuators is selected for the M-FSM: The M-FSM exploits a derivation and an integration of the CTEC MICA ${ }^{\mathrm{TM}}$ based on variable reluctance forces [6] to drive a mobile part in Rx and Ry motions in an efficient way.

Some FSM have already been designed using variable reluctance forces $[7,8]$ to provide improvements above Voice Coil but they exploit attraction forces by reducing the air gap thickness leading to some drawbacks: Contact with attracting poles is responsible for strokes limitations and may generate damages, especially under launching vibrations and shocks. Torques that are not constant versus position are complexifying the drive of such devices.

CTEC M-FSM concept removes these limitations thanks to a derived MICATM concept. MICAs are biased variable reluctance tangential forces actuators. They are interesting in embedded application, such as new space compressors [9]. This space application provides heritage on magnetic components as well as on ultra-long lifetime flexure bearing, useful for space-grade M-FSM.

The MICA ${ }^{\mathrm{TM}}$ stands for Moving Iron Controllable Actuator. The design principle relies on fixed stators which includes the both fixed coils, and fixed permanent magnets. Such design has allowed to achieve optimal thermal draining design of coil heating, with efficient heat sinking by conduction under vacuum, to provide highest temperature stability in space environment. The moving part is built upon a single ferromagnetic piece carrying the SIC mirror and is driven according magnetic reluctance principle, offering very high electrical efficiency and very low Joules effect. The moving part is mounted onto flexure bearings, providing ultra-long fatigue life required for space requirements of nonstop continuous operation at high frequency.

As a result, such MICA FSM produces similar controllable response as Voice Coil based FSM, but with a force-to-mass ratio typically twice larger, with 10 times less heating, and with stable coil temperature. 
The M-FSM are based on 4 MICA-like magnetic actuators.

As for MICA ${ }^{\mathrm{TM}}$ [6], the stator contains the coils and the magnetic poles. These poles are located in front of the moving part. The moving part of the M-FSM exhibits a $32 \mathrm{~mm}$ diameter $17 \mathrm{gr}$ cylindrical magnetic plate. It can be actuated in Rx and Ry rotations when the air gaps between the stator poles and the moving part are energised by feeding the coils of currents. This moving part can tilt inside the stator without air gap thickness reduction but with air gap surface modification.



Figure 3. M-FSM structure (ZX cross section)

As a consequence, angular stroke is not limited by poles contact. Thus, there is no risk to damage the poles or the moving part when launching vibrations and shocks. In addition, torque is rather proportional to applied current and torque versus position is relatively uniform (Figure 4), providing good controllability. Torque constant is $10 \mathrm{mN}$.m/A. The coils are designed to support 10Apk current. A small reluctance torque at rest if tilted ensures an alignment of the moving part with the poles.

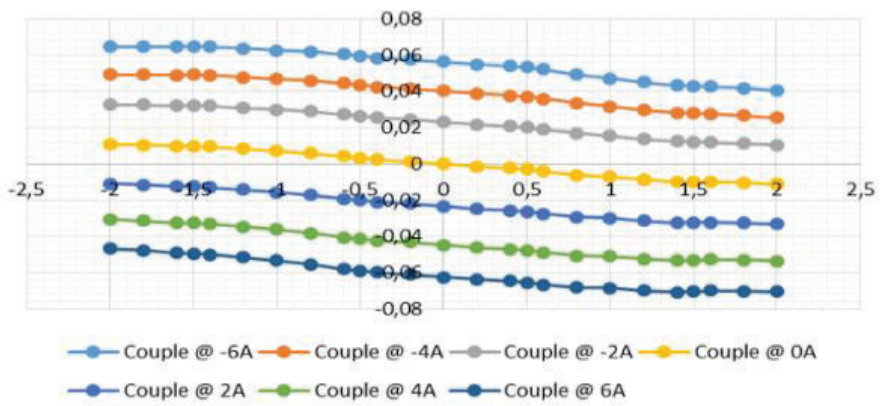

Figure 4. Torque vs angle at different currents

A frictionless flexure bearing guides along Rx and Ry the moving plate on which is fixed a mirror oriented along z. This flexure bearing ensures both high resolution and infinite lifetime. The guiding stiffness is chosen to get the first resonance frequency at $100 \mathrm{~Hz}$. The mirror's diameter can vary from $10 \mathrm{~mm}$ as in the BBM to $31 \mathrm{~mm}$ as in the EM.

To sense the mirror position, the M-FSM contains 4 probes called ECP500 located below the mirror. These ECP500 are small proximity Eddy Current Sensors based on PCB coils, developed by CTEC, spatialised with CNES support and available as standard products. ECP500 can measure up to $1 \mathrm{~mm}$ range with a $3 \mathrm{~nm}$ resolution at $1 \mathrm{kHz}$. Today this $1 \mathrm{~mm}$ range provides the stroke limits of the moving part. Using 4 ECP500 allows to measure Rx and Ry tilts in a differential mode to avoid temperature dependency and integration bias by offset compensations.

These sensors allow a good observability of the mirror position and improve the controllability of the mechanism when working in closed loop. 
Associated electronics for sensing, driving and controlling are the ECS45 conditioner and the MCSA480 driver (Figure 5). The ECS45 is a two channels conditioner reading the 4 ECP500, offering a bandwidth of $20 \mathrm{kHz}$.

The MCSA480 is a new two channels current switching amplifier from CTEC for inducting loads. It is able to provide both $10 \mathrm{~A} \mathrm{AC}$ current and $48 \mathrm{~V} \mathrm{AC}$ voltage per channel. It drives the M-FSM in push-pull mode. Its 10kHz@-3dB bandwidth is compatible with required large bandwidth in closed loop. Today the closed loop control is a PID, offering overall performances after a trade-off of control/drives study. But the implemented controller with very high sampling rate ( $>50 \mathrm{ksamples} / \mathrm{s})$ could support advanced control laws for better performances.

These electronics offer both open \& closed loop control modes with high dynamic performances thanks to high power limits and a fast controller.

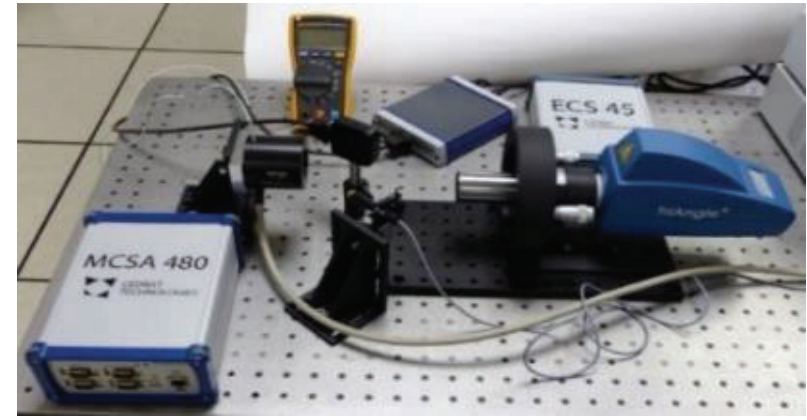

Figure 5. Optical test bench with ECS45 and MCSA480

\section{M-FSM OPEN LOOP PERFORMANCE}

The electromechanical strokes have been measured with a dedicated optical test bench (Figure 5), because there was no standard equipment measuring accurately the $+/-2^{\circ}$ mechanical stroke on a bandwidth up to $1 \mathrm{kHz}$. This combines a TRIOPTICS TriAngle autocollimator and a SITEK PSD. The PSD calibration has been performed using the autocollimator. Then the M-FSM ECP500 sensors have been calibrated using the PSD.

Low level angular strokes vs frequency (Figure 6) shows a constant gain up to the first resonance frequency (100Hz). Comparison of gain and phase of 2 different M-FSM62 for both channels shows a satisfying reproducibility.
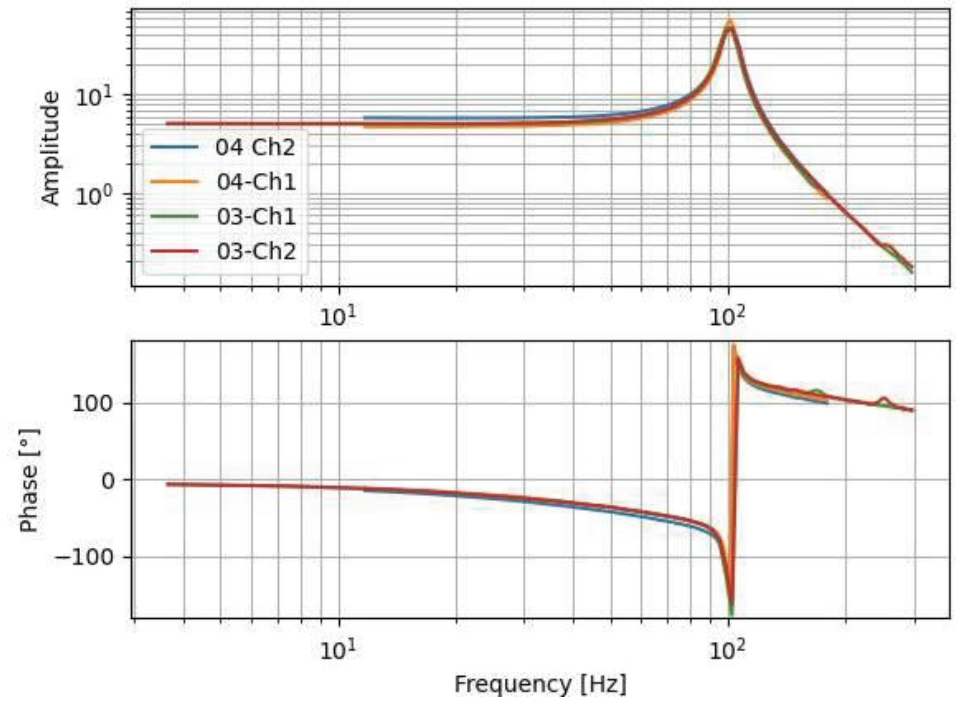

Figure 6. Low level of 2 M-FSM62 bandwidth. 
High level angular stroke has been measured at different frequencies. For example, a measured $+/-35 \mathrm{mrad}$ sine stroke at $20 \mathrm{~Hz}$ is produced with $1.56 \mathrm{Vrms}$ and $2.64 \mathrm{Arms}$ meaning a power of only $3.75 \mathrm{~W}$. The full stroke open-loop is larger than $200 \mathrm{~Hz}$. Experimental functional performances are sump up in table 1.

\begin{tabular}{|l|c|c|}
\hline Parameter & Unit & Value \\
\hline Angular stroke max & $\mathrm{Mrad}$ & $+/-34$ \\
\hline Full Stoke Open Loop & $\mathrm{Hz}$ & 250 \\
\hline Mirror stroke @ $200 \mathrm{~Hz}$ & $\mathrm{Mrad}$ & $+/-34$ \\
\hline 1st resonance frequency & $\mathrm{Hz}$ & 100 \\
\hline Resolution & $\mu \mathrm{rad}$ & $2-5$ \\
\hline Resistance @ $20^{\circ} \mathrm{C}$ (incl. & $\mathrm{Ohm}$ & 0.5 \\
\hline Inductance @ $20^{\circ} \mathrm{C}$ & $\mathrm{mH}$ & 0.64 \\
\hline Max drive voltage & $\mathrm{V}$ & 24 \\
\hline Max drive current & $\mathrm{A}$ & 10 \\
\hline Dimensions & $\mathrm{mm}$ & $\varnothing 62 \mathrm{x}$ \\
\hline Total weight & $\mathrm{gr}$ & 400 \\
\hline
\end{tabular}

Table 1. M-FSM Performances

A thermal analysis has been performed in air for different driving conditions.

At nominal conditions, for full stroke and low AC frequency (typically below $50 \mathrm{~Hz}$ ), because of the low electric power, the steady state of the mechanism is possible without overheating and damages.

Another driving conditions, representing severe conditions at $200 \mathrm{~Hz}$ with $10 \mathrm{Apk}$ to get the max stroke is analysed. The frequency is twice above the resonance frequency explaining the required current. Heating measurement versus time (Figure 7) show that this severe drive condition can be maintained for 10 minutes. In correlation with low power requirement and the coils location in the stator, the thermal heating of the mechanism appears quite limited (lower than $100^{\circ} \mathrm{C}$ at the heat source) and concentrated in the stator. This reduces the heating of the mobile part supporting the mirror. These limits mirror thermal deformation and so keeps the flatness of the mirror.

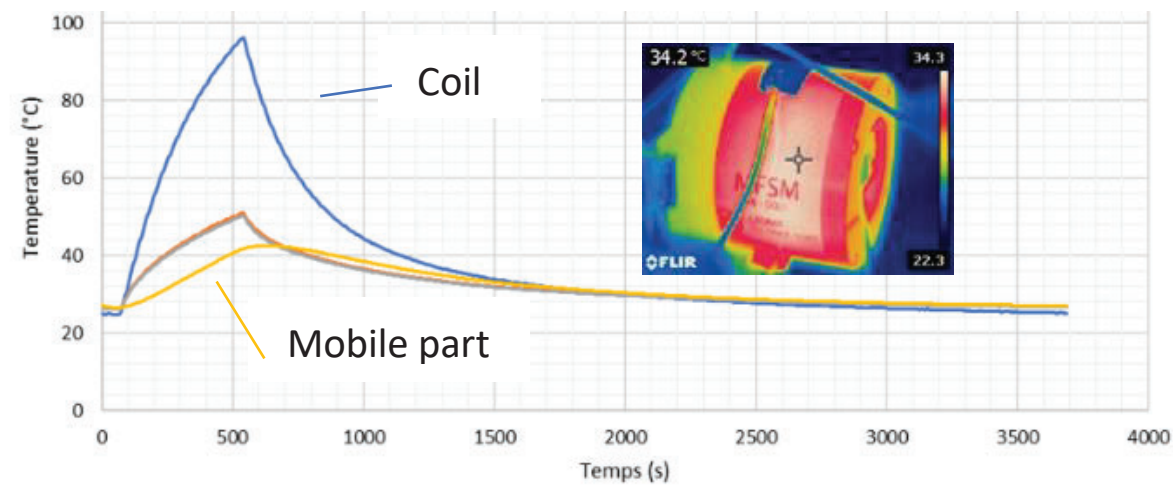

Figure 7. Heating at $10 \mathrm{Apk} 200 \mathrm{~Hz}$ drive

Some vibration analysis have been performed both theoretically and experimentally. In the $[0-2 \mathrm{kHz}]$ bandwidth, only 4 modes have been found and identified by FEM. The vibration tests have been realized with B\&K LDS electrodynamic shaker at CTEC. In these tests, the mirror has been replaced by a lightweight tri-axis accelerometer (0.8g). The MFSM Z-axis excitation shows mainly the pumping mode around $150 \mathrm{~Hz}$ (Figure 8 ). 


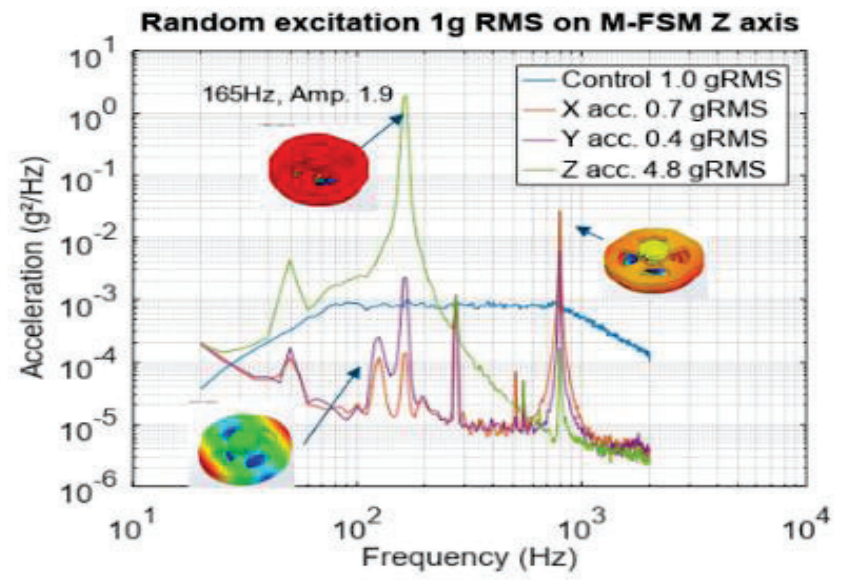

Figure 8. M-FSM Test in random vibration ( $\mathrm{Z}$ axis)

\section{M-FSM CLOSED LOOP CONTROL}

For using the M-FSM in FSO, a closed loop control is required for different functions such as step \& stay positioning, scanning and stabilization.

The selected control strategy is at first meant to compensate some eddy current effects. These introduce some phase shifts between the motion and the order when going up in frequency.

Thus, the approach is based on a state feedback controller as [5] but explicitly integrating the eddy current effect. The observer rebuilds the states that cannot be sensed, and the state feedback controller provides damping to the system.

$$
\begin{array}{rlrl}
A & =\left[\begin{array}{cccc}
0 & 1 & 0 & 0 \\
-k_{t} / J_{t} & -c_{t} / J_{t} & k_{m} / J_{t} & 0 \\
0 & -k_{m} / L_{H} & -R_{f} / L_{H} & R_{f} / L_{H} \\
0 & 0 & R_{f} / L & -\left(R+R_{f}\right) / L
\end{array}\right] & B=\left[\begin{array}{cc}
0 & 0 \\
1 / J_{t} & 0 \\
0 & 0 \\
0 & 1 / L
\end{array}\right] \\
C=\left[\begin{array}{llll}
1 & 0 & 0 & 0 \\
0 & 0 & 0 & 1
\end{array}\right] & D=\left[\begin{array}{ll}
0 & 0 \\
0 & 0
\end{array}\right]
\end{array}
$$

Figure 9. State space representation of the mechanism

This strategy allows to reject the eddy current effect and then achieve efficient damping on the system.

The tuning of a state feedback-based controller is based on the identification of the model of the system, including the eddy current effect. This first step is essential in case of developing model based advanced control (Figure 10).

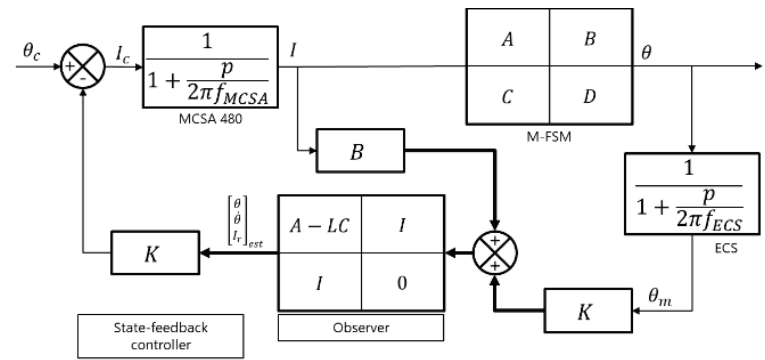

Figure 10. Controller architecture 
The Figure 11 compares the measured low level open loop response with the model. The good correlation of amplitudes and phases shows that the methodology is consistent and thus the model is reliable for control.

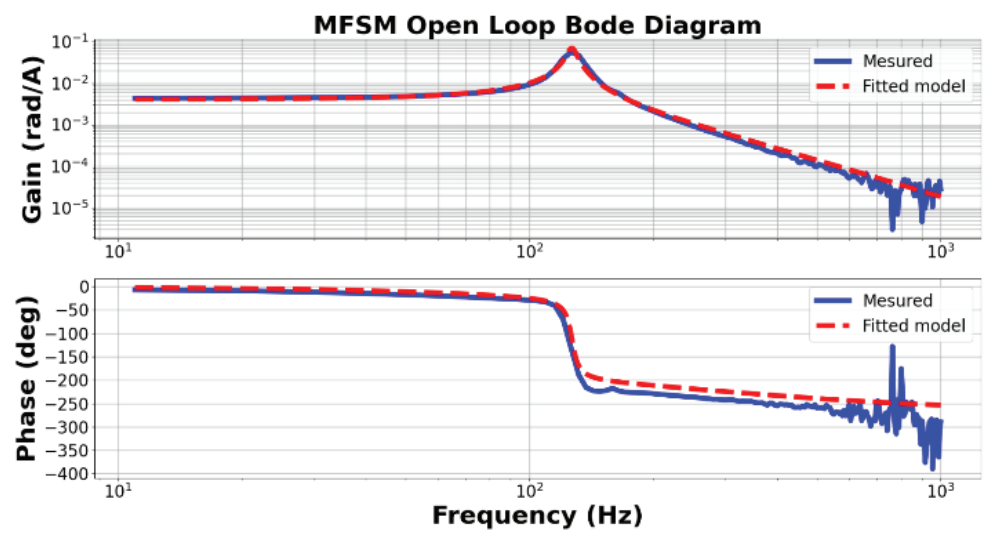

Figure 11. Comparison between model and measurement

The first milestone of the workplan is to control the system slightly above the first resonance frequency $(\sim 150 \mathrm{~Hz})$. The results presented in Figure 12 show the experimental results with first various controller implementations. These are encouraging.

The next step is to achieve $\sim 1 \mathrm{kHz}$ reference tracking bandwidth. Modelling shows a feasibility as in Figure 13 .



Figure 12. Experimental results of the controller (achieved damping)
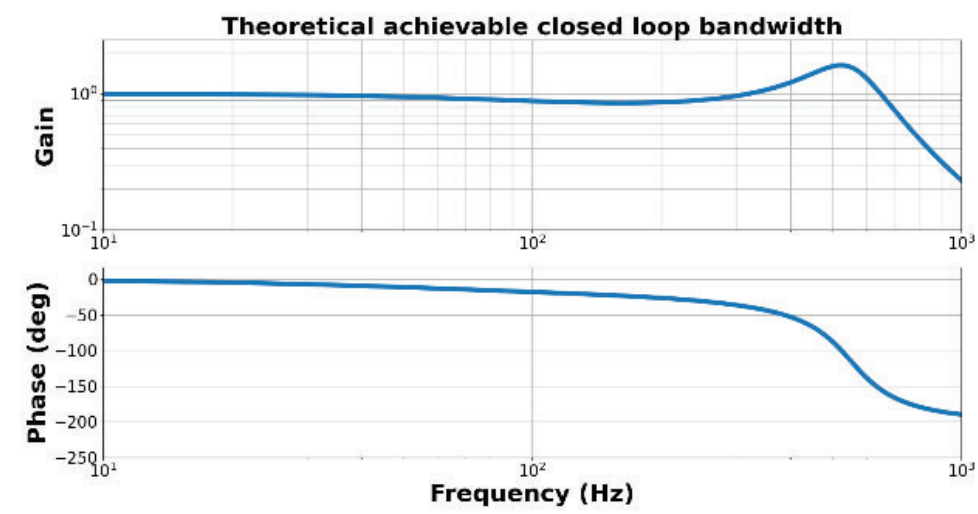

Figure 13. Theoretical achievable closed loop bandwidth 
It is assumed that the final performance will be close to the theory because the previous steps have shown good agreement between the theory and the tests. In addition, very early tests of this control parameters provide good agreement with the figure 13 bandwidth.

\section{CONCLUSION}

New requirements from Space FSO and DSOC have driven CEDRAT TECHNOLOGIES (CTEC) to develop a new type of Fast Steering Mirror mechanism, the M-FSM62, based on magnetic actuation. It targets larger strokes than piezoelectric Tip tilt mechanisms developed by CTEC.

The M-FSM62 takes heritage of CTEC patented MICA TM technology. When tilting a 31mm-diameter mirror, this mechanism offers reproducible Rx Ry strokes $>+/-2^{\circ}$ with a $200 \mathrm{~Hz}$ Full Stroke bandwidth. Requested power is reduced leading to low heating and allowing high duty cycle. Vibration tests allow to define first limits and conditions for the MFSM to manage external vibrations.

Advanced control is achieved using integrated eddy current sensors and a closed loop control based on a state feedbackbased controller. Experimental results show the closed loop control is achieved above the resonance frequency. Modelling and early tests show the feasibility to achieve a bandwidth of some hundred Hertz.

\section{ACKNOWLEDGEMENT}

The authors would like to thank BPI France for its support.

\section{REFERENCES}

[1] R. Le Letty, Miniature Piezo Mechanisms for Optical and Space applications, ACTUATOR Conf, Messe Bremen (G), June 2004, pp 177-180

[2] F. Claeyssen, Beam Steering Mirrors from space applications to optronic solutions, Proc. OPTRO Conf, Paris, Feb. 2018

[3] A. Guignabert, Point Ahead Mechanism for Deep Space Optical Communication - Development of a new PiezoOptical Tip-tilt mechanism, Proc AMS, Houston, May 2020

[4] F. Claeyssen, Large-stroke Fast Steering Mirror for space Free-Space Optical communication, OPTRO 2020, $\mathrm{n}^{\circ} 0062,28-30$ Jan 2020

[5] W. Coppoolse, Dual-axis single-mirror mechanism for beam steering and stabilisation in optical inter satellite links, Proc ESMATS conf, 2003

[6] G. Aigouy, Development of the plain bearing and flexure bearing MICA300CM actuators, Proc. ACTUATOR, Bremen, A.5.5, June 2018, p 150

[7] Y. Long, Modeling and Analysis of a Novel Two-Axis Rotary Electromagnetic Actuator for Fast Steering Mirror, J. of Magnetics 19(2), 2014

[8] Y. Long, Design of a Moving-magnet Electromagnetic Actuator for Fast Steering Mirror, J. of Magnetics, Vol. 19 (3), 2014

[9] G. Aigouy, Zero Boil Off Compressor Based on MICA actuators, ACTUATOR 2021, Mannheim, Feb 2021 